\title{
Clinical Profile and Short-term Outcome of Pediatric Status Epilepticus
}

\author{
MAHESh KAMATE \\ Child Development and Pediatric Neurology Division, Department of Pediatrics, \\ KAHER's JN Medical College, Belgaum, Karnataka 590010. \\ drmaheshkamate@gmail.com
}

S tatus epilepticus (SE) is a common pediatric emergency, yet understanding of contemporary care practices remains incomplete, particularly for children in developing countries. The clinical profile and outcome of SE in children from developing countries is bound to be different than those from the developed countries. But, there are very few studies on pediatric SE from India, especially in the last decade after the new definition of SE was proposed, and many of the published studies have been retrospective studies. The current operational definition of SE is any active seizure of $\geq 5$ minutes duration or recurrent episodes of seizures without gaining consciousness in between that last for more than 5 minutes [1,2]. In this issue of Indian Pediatrics, Chetan, et al. [3] provide some useful prospectively collected data on pediatric SE presenting to a tertiary-care center in India. Using the current operational definition of SE and an objective outcome measure, Pediatric Overall Performance Category, they have described the clinical profile and short-come outcome of 109 children with pediatric SE from January 2017 to April 2018. Their findings provide important information about the etiological profile of SE, the current protocol for management of SE, the response to various anti-epileptic drugs and short-term outcome.

Amongst the various prognostic factors in SE, the duration of convulsive SE is the only modifiable one with proper management. Prolonged seizures are associated with increased risk of mortality and morbidity [4]. Unfortunately, due to lack of public awareness, absence of prompt availability of medical care, and lack of infrastructure to transport to appropriate centers there is significant delay in children reaching the tertiary care centers in developing countries. In the study by Chetan, et al. [3] the average (range) duration of seizure was 17.5 (7-60) min. A shorter interval between onset and initiation of treatment is important for rapid control. However, studies from developing countries have shown that despite significant delay in initiating SE management, the incidence of RSE and case fatality is comparable with other cohorts [5].Prolonged SE or treatment delay should not always be considered a hopeless situation and all cases should be managed aggressively.

The cause of SE is the most important factor that determines morbidity and mortality. Failure to treat the underlying cause promptly and correctly will preclude seizure control regardless of which anti-epileptic drug one chooses. Seizures and brain injury will ensue if we fail to address the underlying hypoglycemia/hypocalcemia or CNS bacterial infection in a patient presenting with refractory seizures and acute encephalopathy [6]. In the current study, $60.6 \%$ children had acute symptomatic etiology and measures to treat the underlying cause need to be instituted along with the management of SE to get good outcome. This fact needs to be stressed in the protocol for management of SE. Infectious etiology for both SE and RSE is common in developing countries like India [5,7].

In the current study, the second dose of midazolam was not effective in any of the children it was administered. It resulted in further delay in administering the second line anti-epileptic drugs. So, this may be taken as evidence for skipping this step in the protocol. Due to rapid internalization of GABA-A receptors, benzodiazepines become rapidly ineffective in management of SE with time [8]. After the first benzodiazepine dose, currently there is insufficient evidence to suggest that one antiepileptic drug is better than the other. Choice can be made based on the cost, availability, past use of that drug and the details of the type of seizures. Intravenous midazolam is the preferred anesthetic agent for management of refractory SE in view of its safety and ease of administration, as was the case in the present study. There is little evidence to choose between midazolam, propofol, and thiopentone, and all have shown comparable efficacy [5].

There is a need for continuous video-EEG monitoring to identify and treat subclinical seizures/SE and to avoid administering potentially harmful anti-seizure medica- 
tions (i.e. barbiturate infusions) to treat clinical paroxysms mimicking seizure [6]. In the current study, EEG monitoring was not done, and this is one of the limitations of the study. Though non convulsive SE is a poor prognostic indicator, whether aggressive treatment of this translates into better outcome is not clear and this is fraught with complications like prolongation of ventilation, hypotension and other medical complications [5].

In the present study [3], non-administration of antiepileptic drugs (AED) prior to presentation to the hospital was found to predict an unfavorable outcome. Preexisting epilepsy was present in around 30\% children. These children are always at a risk of having seizure recurrence. It is important to stress on the drug compliance and prescribe them intranasal/buccal midazolam and train parents how to administer them. A clear plan of treatment depending on the cause of seizures should be given to parents, so that in case of recurrence, appropriate steps can be taken to control the seizures quickly.

In summary, Chetan, et al. [3] provide a much needed insight into current care practices for pediatric SE. Their findings demonstrate the benefits of auditing data to better understand current treatment practices and identify potential areas for refinement. This process helps in the design of both local quality improvement initiatives and future clinical investigations.

Funding: None; Competing interests: None stated.

\section{REFERENCES}

1. Glauser T, Shinnar S, Gloss D, Alldredge B, Arya R, Bainbridge J, et al. Evidence-Based Guideline: Treatment of Convulsive Status Epilepticus in Children and Adults: Report of the Guideline Committee of the American Epilepsy Society. Epilepsy Curr. 2016;16:48-61.

2. Trinka E, Cock H, Hesdorffer D, Rossetti AO, Scheffer IE, Shinnar S, et al. A Definition and Classification of Status Epilepticus- Report of the ILAE Task Force on Classification of Status Epilepticus. Epilepsia.2015; 56: 1515-23.

3. Chetan C, Sharma S, Mathur SB, Jain P, Aneja S. Clinical profile and short-term outcome of pediatric status epilepticus at a tertiary-care center in Northern India. Indian Pediatr. 2020;57:213-17.

4. Eriksson K, Metsaranta P, Huhtala H, Auvinen A, Kuusela AL, Koivikko M. Treatment delay and the risk of prolonged status epilepticus. Neurology.2005;65:1316-8.

5. Hassan H, Rajiv KR, Menon R, Menon D, Nair M, Radhakrishnan A. An audit of the predictors of outcome in status epilepticus from a resource-poor country: A comparison with developed countries. Epileptic Disord. 2016;18:163-72.

6. Payne E. Status epilepticus - The hunt for treatable causes. Semin Pediatr Neurol.2018;26:108-9.

7. Sadik KC, Mishra D, Juneja M, Jhamb U. Clinicoetiological profile of pediatric refractory status epilepticus at a public hospital in India. J Epilepsy Res. 2019;9:36-41.

8. Gainza-Lein M, Fernandez IS, Jackson M, Abend NS, Arya $\mathrm{R}$, Brenton JN, et al. Association of time to treatment with short-term outcomes for pediatric patients with refractory convulsive status epilepticus. JAMA Neurol. 2018;75: 410-8. 